\title{
Pathophysiology of the hypertrophied heart in man*
}

\author{
H. P. Krayenbuehl, O. Hess and H. Hirzel \\ Medical Policlinic, Cardiology, University Hospital, Zürich, Switzerland
}

KEY WORDS: Hypertrophy, left ventricular systolic and diastolic function.

Increase in sympathetic drive, the Frank-Starling effect and myocardial hypertrophy represent the three compensatory mechanisms in chronic mechanical overloading of the heart. Chronic pressure overload is associated with concentric and chronic volume overload with eccentric hypertrophy. The changes in ventricular geometry have an important influence on the ejection dynamics of the heart; the magnitude of fiber shortening is the predominant mechanism for systolic reduction of cavity size in eccentric hypertrophy whereas in concentric hypertrophy the contribution of systolic wall thickening to ejection becomes very important.

The main abnormality of diastolic function in patients with left ventricular $(L V$ ) hypertrophy is the increase of chamber stiffness indicated by the steepened slope of the diastolic pressure-volume relationship. In contrast $L V$ diastolic myocardial stiffness as evaluated from the stress-strain relationship remains relatively unaltered in hypertrophy unless there is massive admixture of fibrosis in the LV wall (congestive cardiomyopathy). Finally LV relaxation (rate of pressure decay) is often impaired in hypertrophied states although the relationship of abnormalities of relaxation to alterations of systolic function remains to be established.

There has been considerable debate whether in secondary $L V$ hypertrophy from chronic pressure or volume overload myocardial contractility is normal or depressed. We have recently shown that in patients with myocardial hypertrophy from aortic stenosis ejection phase indexes of contractility are correlated inversely to peak systolic wall stress and that this relationship is modulated according to the actual inotropic state. The patients on the downward shifted curve (depressed contractile state) had a significantly increased LV angiographic mass. Thus advanced $L V$ hypertrophy in chronic pressure overload appears to be associated with compromised contractile state. The structural and metabolic abnormalities which may be ultimately responsible for the depression of contractility of the hypertrophied human myocardium encompass the following findings: reduced intracellular volume fraction of myofibrils; massive increase of the average fiber diameter and increased variability of the thickness of the individual fibers and reduced activity of the myofibrillar ATPase.

When a prolonged mechanical load is imposed to the heart the acutely mobilizable compensatory mechanisms namely the Frank-Starling effect and increased sympathetic drive rapidly become insufficient for maintaining adequate pump function. In this situation myocardial hypertrophy must develop in order to prevent imminent catastrophe. The mechanism by which hypertrophy (thickening of the ventricular wall) exerts its hemodynamically beneficial effect consists, according to the law of Laplace, in a reduction of the critically elevated wall stress. Furthermore the de-

Requests for reprints 10: H. P. Krayenbuehl, M.D., Medical Policlinic, Cardiology, University Hospital, CH-8091 Zürich, Switzerland. *Supported by a grant from the Swiss National Science Founda-
tion. crease in wall stress allows the augmented oxygen consumption per gram muscle to return to normal values. Thus the development of hypertrophy serves to restore adequate pump function and to normalize energy expenditure per unit mass of muscle in the face of chronic abnormal loading.

Concentric and eccentric hypertrophy. Implications of the ventricular geometry

Traditionally a given type of chronic abnormal loading has been associated with a specific shape of the hypertrophied ventricle ${ }^{[1]}$. Whereas chronic pressure overload leads to concentric hypertrophy characterized by a reduced chamber radius ( $r$ )/wall thickness (h) ratio eccentric hypertrophy is the result of long- 


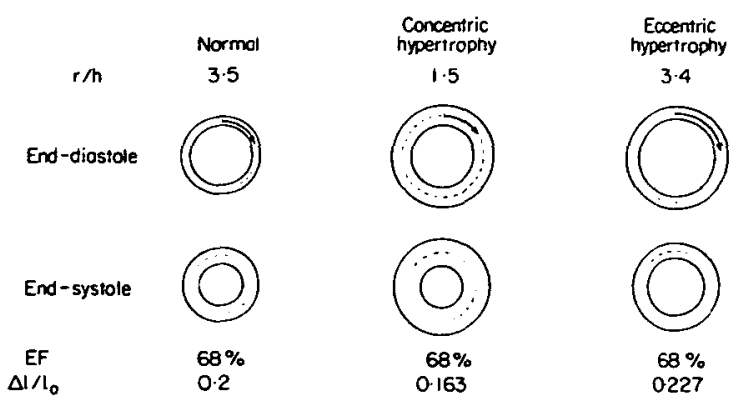

Figure 1 Extent of systolic midwall shortening in a spherical model of ventricles with an identical ejection fraction (EF). Note that for the given EF the ratio of midwall fiber shortening to initial fiber length $\left(\triangle 1 / 1_{0}\right)$ is larger in eccentric than in concentric hypertrophy. In the latter thickening becomes important for the systolic reduction of cavity size. Abbreviations: $r=$ end-diastolic inner chamber radius; $h=$ end-diastolic wall thickness; $\Delta l=$ extent of systolic midwall fiber shortening; $l_{0}=$ end-diastolic midwall fiber length.

standing volume overload. In the compensated stage the increase in $h$ is proportional or almost so to the increase of $r$. Thus the ratio $r / h$ is essentially normal.

The changes in ventricular geometry consequent to chronic abnormal loading are of importance for the ejection dynamics of the heart. Let us assume (Fig. 1) that the ejection fraction is identical in a normal ventricle, a ventricle with concentric and a ventricle with eccentric hypertrophy. As shown in this spherical model ${ }^{[2]}$ the same ejection fraction is obtained by a clearly different fraction of midwall fiber shortening compared to initial fiber length $\left(\triangle 1 / 1_{0}\right)$. Thus in eccentric hypertrophy the magnitude of fiber shortening is the predominant mechanism for systolic reduction of cavity size whereas in concentric hypertrophy the contribution of wall thickening to inward wall displacement (reduction in cavity size) becomes more important and allows for a smaller ratio $\Delta 1 / 1_{0}$ without a fall in ejection fraction.

\section{Chamber and myocardial stiffness in ventricular hyper- trophy}

Patients with different types of hypertrophy elicit different left ventricular pressure-volume (P.V) or dimension (M) loops. Figure 2 depicts some typical P-M curves. It is obvious that not only the systolic portions of the loops vary greatly but also the diastolic P-M relationship is different.

If it is assumed that the diastolic P-V relationship is monoexponential, the constant of chamber stiffness

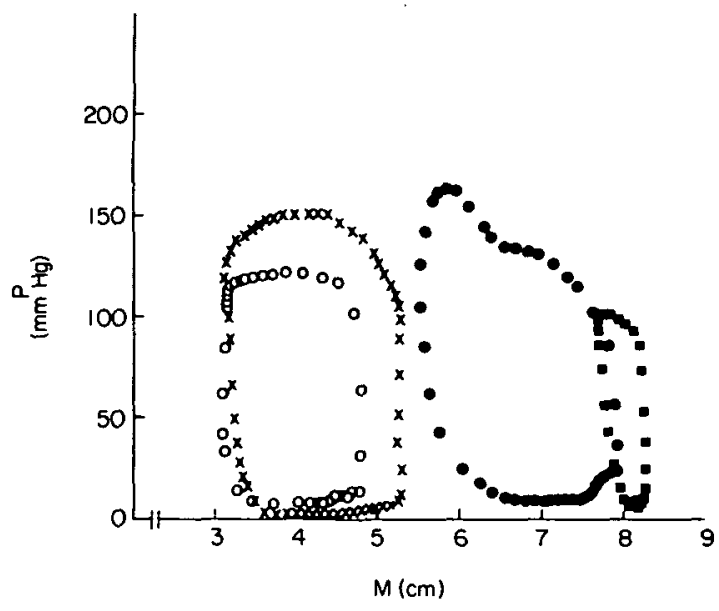

Figure 2 Left ventricular pressure-dimension loops in four patients. Abbreviations: $\mathbf{P}=$ left ventricular pressure; $\mathbf{M}=$ left ventricular transverse diameter determined by single beam echocardiography; $\circ=$ normal subject; $\square=$ congestive cardiomyopathy; $\mathrm{X}=$ aortic stenosis; $\bullet=$ aortic insufficiency.

$\left(k_{p}\right)$ can be derived from the slope of the linear relation between volume stiffness $(\mathrm{dP} / \mathrm{dV})$ and pressure $^{[3]}$. This constant has been shown to be increased in patients with concentric hypertrophy ${ }^{[3]}$. Such an increase of $k_{p}$ is either the consequence of the increase of wall thickness alone or might also be due to changes of the diastolic properties (stiffening) of the muscle itself. The calculation of the constant of muscle stiffness from the left ventricular diastolic stress-strain relationship helps elucidate the nature of a steepened diastolic P-V relationship in more detail. In Fig. 3 left ventricular meridional wall stress $(S)$ is plotted against normalized midwall strain $\left(E_{n}\right)^{[4]}$ in the same four patients depicted in Fig. 2. Whereas the patients with aortic stenosis and aortic insufficiency show a similar curve as the control subject the curve of the patient with congestive cardiomyopathy is sizably steepened. Thus secondary hypertrophy is accompanied by no or minor changes of left ventricular muscle stiffness whereas in the primary hypertrophy of congestive cardiomyopathy the left ventricular diastolic muscular properties are clearly altered. In 40 patients with myocardial hypertrophy we have recently shown ${ }^{[5]}$ that an increased normalized viscoelastic constant of myocardial stiffness $\left(K_{n}\right)$ is associated with increased interstitial fibrosis as evaluated from endomyocardial biopsies (Fig. 4). In contrast cellular hypertrophy estimated from myocardial fiber diameter and left ventricular chamber hypertrophy evaluated from angiographic mass did 
not correlate with $\mathbf{K}_{\mathbf{n}}$. Thus abnormalities of diastolic myocardial stiffness in myocardial hypertrophy appear to be related more to the interstitial than to the muscular tissue.

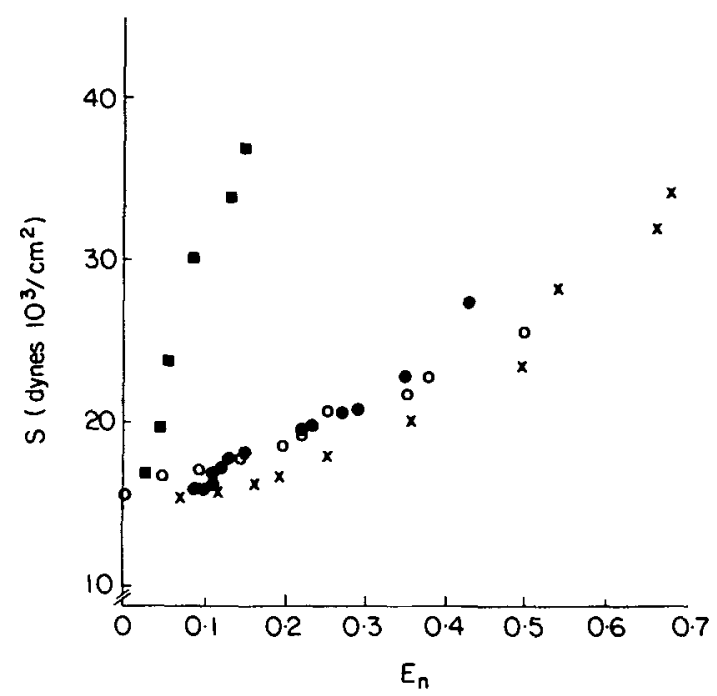

Figure 3 Left ventricular diastolic stress-strain relationship in the same four patients as in Fig. 2. Normalization of the stress-strain data was carried out by using a diastolic circumferential reference length at a common wall stress of 15 dynes $10^{3} / \mathrm{cm}^{2[4]}$. Note that only the patient with congestive cardiomyopathy showed a steepened slope of the stress-strain relationship whereas the slopes of the two patients with aortic valve disease did not differ from that of the control subject. $S=$ meridional wall stress; $E_{n}=$ normalized midwall strain. Symbols as in Fig. 2.

Figure 4 Left ventricular systolic and diastolic myocardial stiffness, angiographic muscle mass, muscle fiber diameter and interstitial fibrosis in eight control subjects $(\mathrm{CO}), 10$ patients with aortic stenosis (AS), 10 patients with aortic insufficiency (AI) and 12 patients with congestive cardiomyopathy (COCM).

The constant of (systolic) series elasticity (k) was approximated by the formula:

$$
\left.\mathrm{k} \text { (circumferences }{ }^{-1}\right)=\frac{0.9 \max \mathrm{dS} / \mathrm{dt}}{\mathrm{V}_{\mathrm{CF}} \text { Speak }}
$$

where $S=$ meridional wall stress; $V_{C F}=$ midwall fiber (contractile element) velocity of shortening at Speak; Speak $=$ peak systolic stress.

The normalized visco-elastic constant of (diastolic) parallel elasticity $\left(\mathrm{K}_{\mathrm{n}}\right)$ was calculated as reported previously ${ }^{[5]}$.

Note that in COCM there was a significant increase of the constant of series and parallel elasticity associated with a sizable increase in interstitial fibrosis. The elasticity constants correlated however neither with the angiographic muscle mass nor with the muscle fiber diameter. $(\mathrm{Bar}=$ mean \pm 1 s.e.m.).
In the course of these studies it has become evident that the stress-strain relationship is often not truly exponential, especially in patients with aortic insufficiency. The observed deviation from an exponential curve in the early part of passive diastolic filling has been attributed to visco-elastic influences and hence linearization of the $\ln$ stress-strain relationship was obtained by accounting for individual strain rates ${ }^{[4]}$. However it must be realized that an alternative mechanism which could also lead to the observed early deviation from a true exponential is a prolongation of relaxation in left ventricular hypertrophy.

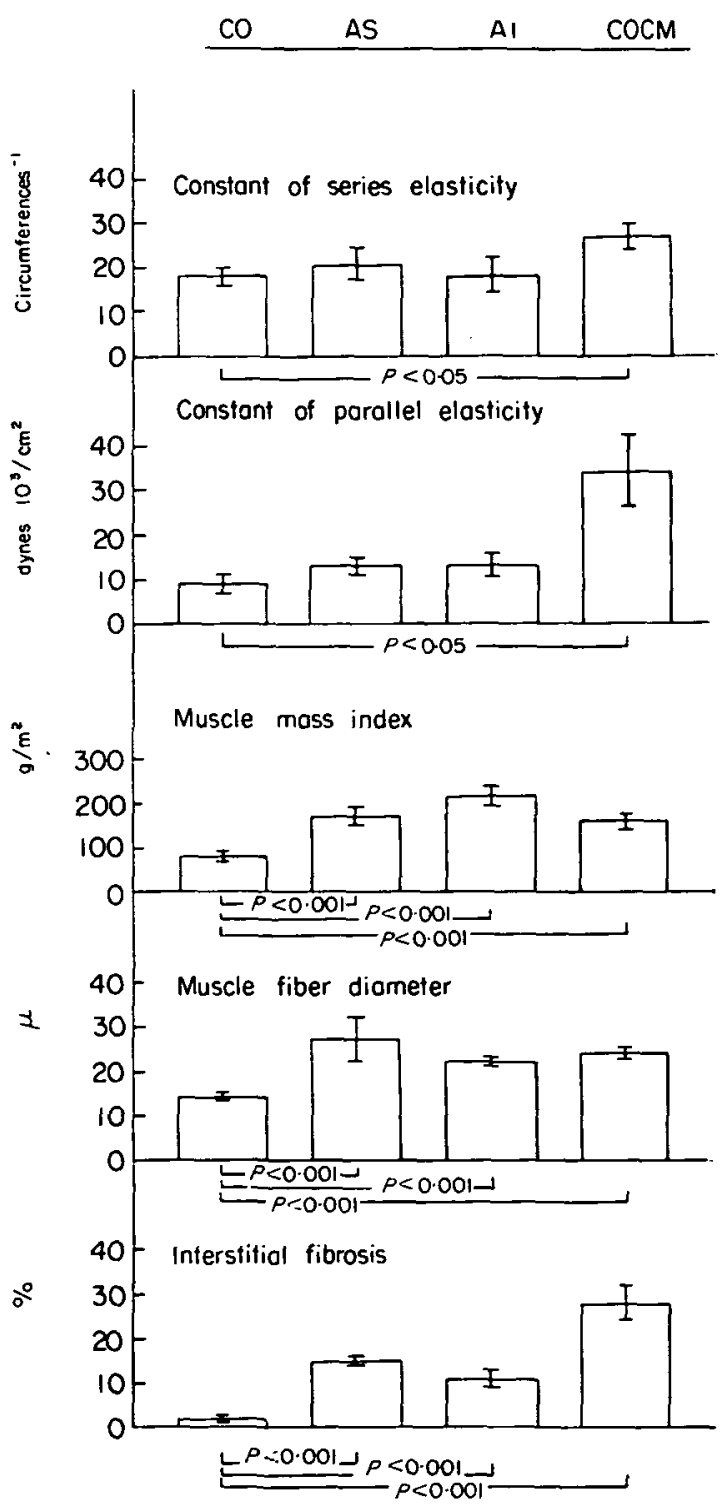




\section{Relaxation in ventricular hypertrophy}

There are few data in the literature on left ventricular relaxation in hypertrophic states in man. In patients with aortic stenosis delayed mitral valve opening has been reported ${ }^{[6]}$ and in hypertrophic cardiomyopathy an increased time constant of isovolumic left ventricular pressure fall as evaluated from the quotient $P_{\text {at peak neg dP/dt/peak neg } \mathrm{dP} / \mathrm{dt} \text { was }}$ found ${ }^{[7]}$. We have recently assessed relaxation from simultaneous left ventricular pressure-single beam echo measurements in 58 patients with secondary hypertrophy from aortic valve disease (23 AS, 18 combined lesion, $17 \mathrm{AI}$ ) and in 10 controls $^{[8]}$. Peak

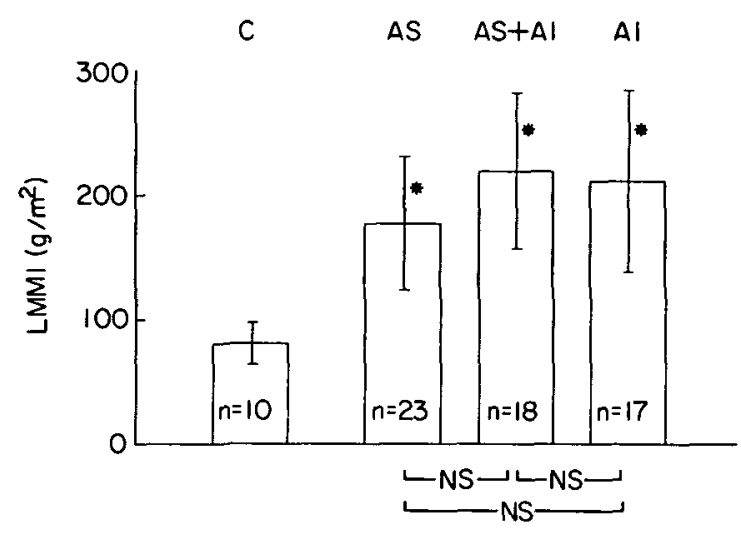

Figure 5 Angiographic muscle mass (LMMI) in 68 patients in whom left ventricular relaxation dynamics were evaluated. Abbreviations: $\mathrm{C}=$ controls; $\mathrm{AS}=$ aortic stenosis; $\mathrm{AS}+\mathrm{Al}$ $=$ combined aortic valve lesion; $\mathrm{AI}=$ aortic insufficiency; $\mathrm{n}=$ number of patients; NS $=$ not significant. ${ }^{*} P<0.01$ compared to controls. $(\mathrm{Bar}=$ mean \pm 1 s.d.).

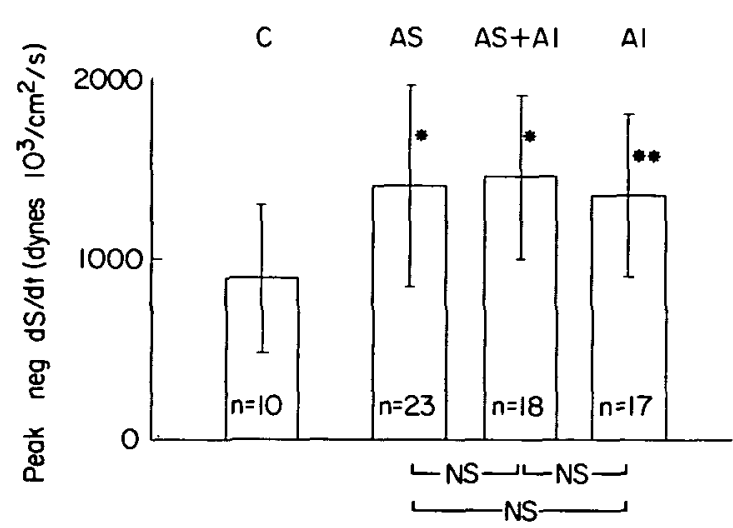

Figure 6 Peak rate of fall of left ventricular meridional wall stress (peak neg $d S / d t$ ) in the same 68 patients as in Fig. 5. Note that peak neg dS dt was increased significantly in all three groups with aortic valve disease. Abbreviations as in Fig. 5. ${ }^{*} P<0.01$ : $^{* *} P<0.02$ compared to controls. rate of fall of left ventricular pressure (peak neg $\mathrm{dP} / \mathrm{dt}$ ) and the time constant $(\mathrm{T})$ of the exponential pressure fall during the interval $A_{2}$ to mitral valve opening were used as indexes of relaxation of the left heart chamber whereas peak rate of fall of meridional wall stress (peak neg $d S / d t$ ) was considered to be an index of left ventricular myocardial relaxation. Left ventricular hypertrophy as estimated from the angiographic muscle mass occurred in all three groups with aortic valve disease. The largest mass was present in the patients with combined lesions (Fig. 5). Left ventricular peak neg $\mathrm{dP} / \mathrm{dt}$ was not different in the three groups with aortic valve disease as compared to the control subjects; peak neg dS/dt was however significantly increased in all three groups with hypertrophy (Fig. 6). It is obvious that from these isolated parameters no clear-cut conclusion about the inherent speed of myocardial relaxation can be drawn because peak neg $\mathrm{dP} / \mathrm{dt}$ is also influenced by peak systolic pressure, and peak neg $\mathrm{dS} / \mathrm{dt}$ by peak systolic stress ${ }^{[9]}$. Thus peak neg $\mathrm{dP} / \mathrm{dt}$ was compared with left ventricular peak systolic pressure. In all three groups with aortic valve disease the significantly increased peak pressure was not accompanied by an increased peak neg $\mathrm{dP} / \mathrm{d}$. This observation suggests a relative depression of peak neg $\mathrm{dP} / \mathrm{dt}$ in these patients. In contrast the increased left ventricular peak stress was associated with a commensurately increased peak neg $\mathrm{dS} / \mathrm{dt}$. The time constant of left ventricular pressure decline was significantly increased in all three groups with hypertrophy (Fig. 7). These observations suggest that the speed of left heart chamber relaxation

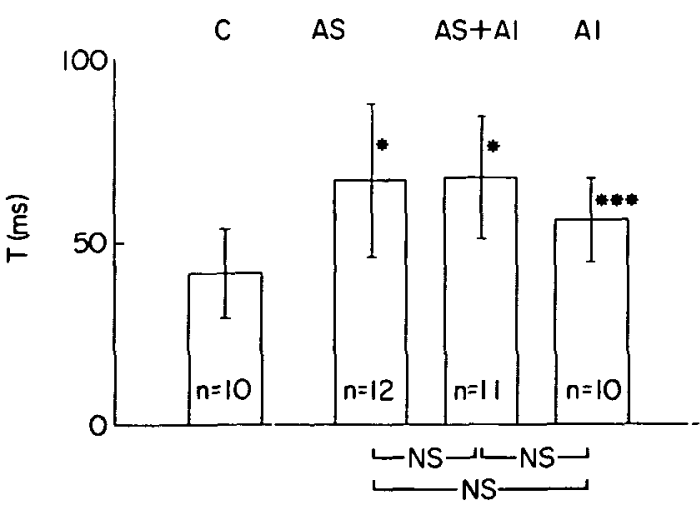

Figure 7 Time constant ( $\mathrm{T}$ ) of left ventricular pressure fall during the early relaxation period $\left(\mathrm{A}_{2}\right.$ to mitral valve opening) in 43 of the 68 patients of Fig. 5 with clearly visible $A_{2}$ and early diastolic separation of the mitral leaflets. $T$ was prolonged in all three groups with hypertrophy from aortic valve disease. Abbreviations as in Fig. 5 . ${ }^{*} P<0.01 ;{ }^{* *}<0.05$ compared to controls. 
is impaired in secondary hypertrophy whereas the rate of myocardial relaxation seems to be essentially unaltered. Admittedly these results appear to be somewhat conflicting. It is to be hoped that an analysis of the entire time course of stress decay will shed more light on the nature of myocardial relaxation in hypertrophy. Furthermore it is not known whether relaxation disturbances in hypertrophy are sufficiently marked to influence left ventricular chamber and muscle dynamics during the period of 'passive' diastolic filling, and whether relaxation disturbances occur in conjunction with contraction abnormalities or are independent of systolic function.

\section{Contractile state of the hypertrophied myocardium}

There has been considerable debate whether in secondary left ventricular hypertrophy from chronic pressure or volume overload myocardial contractile function is normal or depressed. A major part of this controversy is due to the fact that there is no unanimously accepted technique for quantitating left ventricular contractility under all hemodynamic circumstances. The popular ejection phase indices may be inadequate for the evaluation of inotropic state in the presence of an increased afterload. In this context it has been suggested by Gunther and Grossman $^{[10]}$ that in severe aortic stenosis afterload mismatch may be such as to depress left ventricular ejection fraction down to the extreme of $19 \%$. Nevertheless impaired contractility can also be at the origin of a depressed ejection fraction in aortic stenosis ${ }^{[1]}$. We have recently analysed the determinants of left ventricular ejection performance in 76 patients with

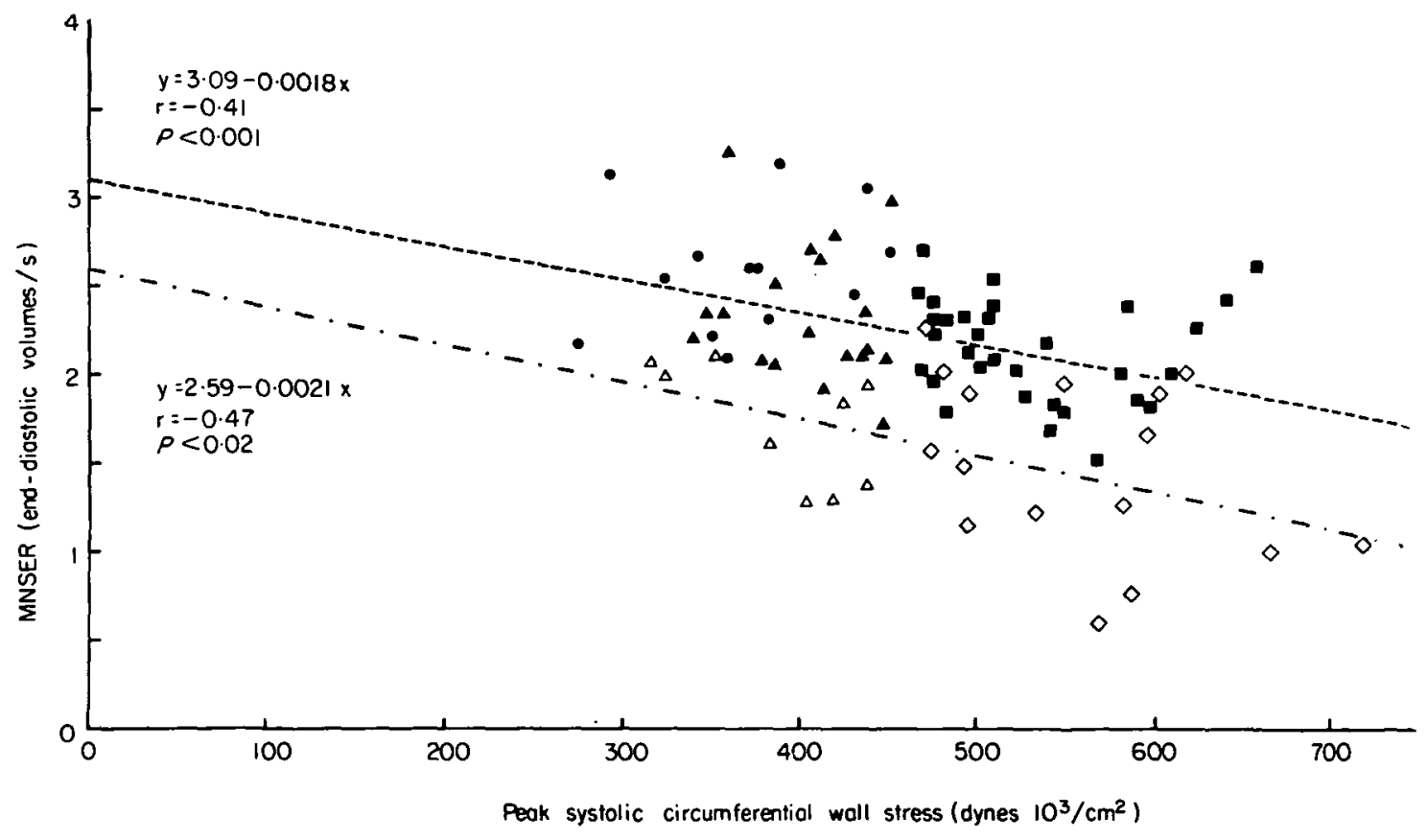

Figure 8 Relationship between normalized systolic ejection rate (MNSER) and peak systolic circumferential wall stress in patients with aortic stenosis.

Separate linear regression analysis was performed for the patients with normal contractility (closed symbols) and for the patients with depressed contractility (open symbols). Groups I and HI represent patients with aortic stenosis having a normal peak systolic wall stress and either normal (closed triangles) or depressed (open triangles) isovolumic indices Groups II and IV represent patients with aortic stenosis and increased peak systolic wall stress having either normal (closed squares) or depressed (open squares) isovolumic indices. $\mathrm{CO}=$ control subjects (dots). Group 1, $\mathrm{n}=19$; Group II, $\mathrm{n}=32$; Group III, $\mathrm{n}=9$; Group IV, $\mathrm{n}=16$; control suhjects, $\mathrm{n}=13$.

Both regressions show a significant inverse relationship between MNSER and peak systolic wall stress. Comparison of the two lines by covariance regression analysis yielded no significant difference for the slopes but a significantly $(P<0.001)$ higher intercept in the patients with normal isovolumic contractility $(3.09$ end-diastolic volumes/s) than in those with depressed contractility ( 2.59 end-diastolic volumes/s). Thus, MNSER depended on both contractile state and afterload. Reprinted with the permission of Huber and coworkers ${ }^{[12]}$. 
pure or predominant aortic stenosis ${ }^{[12]}$. Like Gunther and Grossman ${ }^{[10]}$ we found an inverse relationship between mean normalized systolic ejection rate (MNSER; i.e. ejection fraction divided by ejection time) and peak systolic circumferential wall stress (Fig. 8). This relationship was, however, modulated according to the actual inotropic state, evaluated from pressure-derived velocity indices, since the curve of the patients with depressed velocity indexes was shifted downwards compared to the curve of the patients with velocity indices within the normal range. Our observation that angiographic muscle mass, which represents an index for chamber hypertrophy, was significantly $(P<0.005)$ increased $(197$ $\mathrm{g} / \mathrm{m}^{2}$ ) in the 25 patients with depressed contractility (dashed-dotted curve in Fig. 8) as compared to the 51 patients $\left(157 \mathrm{~g} / \mathrm{m}^{2}\right)$ with preserved contractile state (dotted line in Fig. 8) is relevant to the discussion of whether contractility is depressed in pressure overload hypertrophy from aortic stenosis. We concluded that in chronic pressure overload advanced hypertrophy is associated with depressed contractile state.

Another important issue which has been debated in the past is whether a given degree of increase of left ventricular mass has different consequences for myocardial contractility when hypertrophy is produced by pressure, volume or mixed ventricular overload. In the animal model ${ }^{[13,14]}$ chronic volume overload was reported to have little or no detrimental effect on left ventricular contractile function whereas in man with volume or mixed overload from aortic insufficiency depression of contractile function is not uncommon $^{[15-17]}$. We have recently addressed this question in 130 patients with aortic valve disease and 13 control subjects studied by left ventricular micromanometry and quantitative cine-angiography ${ }^{[18]}$. There were 42 patients with AS, 50 with a combined lesion and 38 with $\mathrm{Al}$. These three groups were subdivided into patients with moderate increase in angiographic mass (125 to $179 \mathrm{~g} / \mathrm{m}^{2}$, Group I) and in those with severe increase in mass $\left(\geqslant 180 \mathrm{~g} / \mathrm{m}^{2}\right.$, Group II). Left ventricular contractile state was assessed by plotting left ventricular isovolumic maximal rate of rise of circumferential wall stress against end-diastolic wall stress. For the calculation of maximal rate of stress rise it was assumed that left ventricular dimensions do not change during the isovolumic contraction period. Making this assumption the maximal rate of rise of wall stress can be determined from max $\mathrm{dP} / \mathrm{dt}$ and left ventricular enddiastolic dimensional measurements. In this 'isovolumic' ventricular function diagram all groups with left ventricular hypertrophy were located rightward

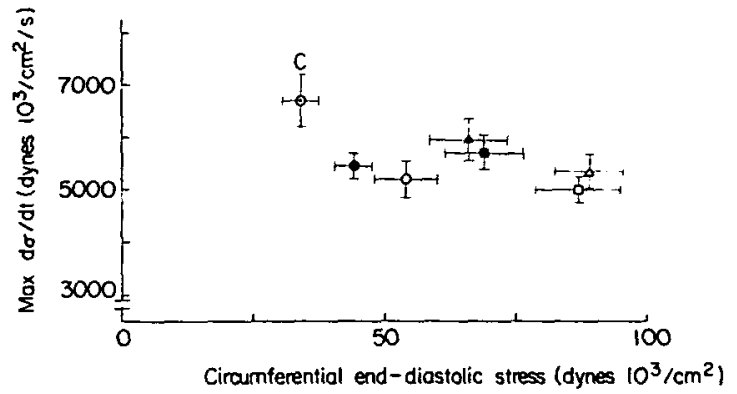

Figure 9 Left ventricular isovolumic maximal rate of rise of circumferential wall stress (vertical axis) $v$. circumferential end-diastolic wall stress (horizontal axis).

The mean values \pm 1 standard error (s.e.) of the six groups of aortic valve disease and of the control group (C) are plotted. Note that all groups with left ventricular hypertrophy were located rightward and downward to the control group eliciting less isovolumic performance at an increased preload. This pattern is indicative of impaired left ventricular contractile function. $\mathrm{AS}=$ aortic stenosis; $\mathrm{AS}+\mathrm{AI}=\mathrm{com}-$ bined aortic valve lesion; $\mathbf{A I}=$ aortic insufficiency; Group I, left ventricular (LV) angiographic mass $<180 \mathrm{~g} / \mathrm{m}^{2}$; Group II, LV angiographic mass $\geqslant 180 \mathrm{~g} / \mathrm{m}^{2}$. AS: $\bullet=$ Group I, $\circ=$ Group II; AS + AI: $\square=$ Group I, $\mathrm{Q}=$ Group II; AI. $\Delta=$ Group I, $\Delta=$ Group II. Reprinted with permission of Krayenbuehl and coworkers ${ }^{[18]}$.

and downward of the control group. This indicates reduced isovolumic performance at an increased preload (Fig. 9), and reveals impaired left ventricular contractile function. At both a moderately and a severely increased mass the patients with combined lesions and with aortic insufficiency had more severely depressed contractile function than the patients with aortic stenosis. We can conclude from these investigations that at a similarly increased left ventricular angiographic mass contractile function appears to be more impaired in eccentric ( $\mathrm{AI}, \mathrm{AS}+\mathrm{AI})$ than in concentric hypertrophy (AS).

\section{Factors responsible for depressed function in myo- cardial hypertrophy}

In man there is increasing interest in the correlation of findings obtained from endomyocardial biopsies with functional parameters. Schwarz and coworkers ${ }^{[19]}$ have reported that in patients with decompensated pressure overload from aortic stenosis the intracellular volume fraction of myofibrils is reduced compared to AS patients with preserved ejection performance. One may speculate whether this structural abnormality was the underlying cause for the depressed contractile function. In patients with aortic 
insufficiency, we have recently analysed ${ }^{[20]}$ whether the prognostically unfavorable combination of reduction of left ventricular minor equator shortening below $25 \%$ and of an increase of the end-systolic minor equator of more than $55 \mathrm{~mm}^{[17]}$ is associated with morphometric alterations. Patients presenting with this particular combination of parameters had a significantly larger mean myocardial fiber diameter, a larger intrapatient variability of fiber diameters and more ultrastructural cellular abnormalities than patients with both or at least one of the hemodynamic measures within normal limits. Moreover we have shown that pre-operative excessive fiber thickening in patients with aortic valve disease heralds an unfavorable postoperative outcome, regardless of the pre-operative left ventricular muscle mass ${ }^{[21]}$. Finally enzymic analysis of left ventricular biopsy samples in patients with valvular disease has shown a significant reduction of myofibrillar ATPase activity when left ventricular function was poor ${ }^{[22]}$. Hopefully in the future the structural and the biochemical evaluation of biopsies can be further refined to give more complete insight into the basic mechanisms responsible for myocardial dysfunction in hypertrophy.

Hypertrophy, like so many things in life, has contradictory aspects ${ }^{[23]}$. Hypertrophy is necessary in order to prevent a catastrophe, and therefore is beneficial in the presence of severe loading. However, this compensatory mechanism has inherent dangers for the proper function of the individual muscular units, and thus in some respects hypertrophy is also detrimental.

\section{References}

[1] Grossman W. Cardiac hypertrophy: useful adaptation or pathologic process? Am J Med 1980; 69: 576-84.

[2] Kreuzer H, Neuhaus KL. Invasive Untersuchungsmethoden zur Erfassung einer gestörten Kontraktilität Verh Dtsch Ges Kreislaufforschg 1976; 42: 31-9.

[3] Gaasch WH, Levine HJ, Quinones MA, Alexander JK. Left ventricular compliance: mechanisms and clinical implications. Am J Cardiol 1976; 38: 645-53.

[4] Hess OM, Grimm J, Krayenbuehl HP. Diastolic simple elastic and viscoelastic properties of the left ventricle in man. Circulation 1979; 59: 1178-87.

[5] Hess OM, Schneider J, Koch R, Bamert C, Grimm $\mathrm{J}, \mathrm{Krayenbuehl}$ HP. Diastolic function and myocardial structure in patients with myocardial hypertrophy. Circulation 1981; 63: 360-71.

[6] Gibson G, Traill TA, Hall RJC, Brown DJ. Echocardiographic features of secondary left ventricular hypertrophy. Br Heart J 1979; 41: 54-9.

[7] Hirota Y. A clinical study of left ventricular relaxation. Circulation 1980; 62: 756-63.
[8] Eichhorn P, Grimm J, Koch R, Hess O, Carroll J, Krayenbuehl HP. Left ventricular relaxation in secondary hypertrophy from aortic valve disease. Circulation (in press).

[9] Weisfeldt ML, Scully HE, Frederiksen J, Rubenstein JJ, Pohost GM, Beierholm E, Bello AG, Daggett WM Hemodynamic determinants of maximum negative $\mathrm{dP} /$ $\mathrm{dt}$ and periods of diastole. Am J Physiol 1974; 227: 613-21.

[10] Gunther S, Grossman W. Determinants of ventricular function in pressure-overload hypertrophy in man. Circulation 1979; 59: 679-88.

[11] Carabello BA, Green LH, Grossman W, Cohn LH, Koster JK, Collins JJ Jr. Hemodynamic determinants of prognosis of aortic valve replacement in critical aortic stenosis and advanced congestive heart failure. Circulation 1980; 62: 42-8.

[12] Huber D, Grimm J, Koch R, Krayenbuehl HP. Determinants of ejection performance in aortic stenosis. Circulation 1981; 64: 126-34.

[13] Taylor RR, Covell JW, Ross J Jr. Left ventricular function in experimental aorto-caval fistula with circulatory congestion and fluid retention. $J$ Clin Invest 1968; 47: 1333-42.

[14] Cooper G, Puga FJ, Zujko KJ, Harrison CE, Coleman HN. Normal myocardial function and energetics in volume-overload hypertrophy in the cat. Circ Res 1973; 32: $140-8$.

[15] Krayenbuehl HP, Turina M, Hess OM, Rothlin M, Senning $\AA$. Pre- and postoperative left ventricular contractile function in patients with aortic valve disease. Br Heart J 1979; 41: 204-13.

[16] Clark DG, McAnulty JH, Rahimtoola SH. Valve replacement in aortic insufficiency with left ventricular dysfunction. Circulation 1980; 61: 411-21.

[17] Henry WL, Bonow RO, Borer JS, Ware JH, Kent KM, Redwood DR, McIntosh CL, Morrow AG, Epstein SE. Observations on the optimum time for operative intervention for aortic regurgitation, I. Evaluation of the results of aortic valve replacement in symptomatic patients. Circulation 1980; 61: 471-83.

[18] Krayenbuehl HP, Huber D, Koch R, Turina M, Senning $\AA$. Contractilité du ventricule gauche hypertrophié par suite d'une lésion de la valvule aortique. Coeur 1980 11: 429-39.

[19] Schwarz F, Schaper J, Kittstein D, Flameng W, Walter P, Schaper W. Reduced volume fraction of myofibrils in myocardium of patients with decompensated pressure overload. Circulation 1981; 63: 1299-304.

[20] Schneider J, Eichhorn P, Tsuruta U, Krayenbühl HP. Correlation between prognostic hemodynamic parameters and myocardial structure in aortic insufficiency. Eur Heart J 1981; 2 (suppl A): 96. (Abstract).

[21] Krayenbuehl HP, Schneider J, Turina M, Senning Å. Myocardial function and structure in aortic valve disease before and after surgery. Circulation 1980; 62: III-207. (Abstract).

[22] Peters TJ, Brooksby IAB, Webb-Peploe MM, Wells G, Jenkins BS, Coltart DJ. Enzymic analysis of cardiac biopsy material from patients with valvular heartdisease. Lancet 1976; 1: 269-70.

[23] Krayenbuehl HP. Effects of hypertrophy on contractile function in man. Basic Res Cardiol 1977; 72: 1849. 
